\title{
Tunneling spectroscopy of the superconducting state of $\mathrm{URu}_{2} \mathrm{Si}_{2}$
}

\author{
A. Maldonado, ${ }^{1}$ I. Guillamon, ${ }^{1}$ J. G. Rodrigo, ${ }^{1}$ H. Suderow,,${ }^{1, *}$ S. Vieira, ${ }^{1}$ D. Aoki, ${ }^{2}$ and J. Flouquet ${ }^{2}$ \\ ${ }^{1}$ Laboratorio de Bajas Temperaturas, Departamento de Física de la Materia Condensada, Instituto de Ciencia de Materiales Nicolás \\ Cabrera, Facultad de Ciencias, Universidad Autónoma de Madrid, 28049 Madrid, Spain \\ ${ }^{2}$ INAC, SPSMS, CEA Grenoble, 38054 Grenoble, France
}

(Received 20 December 2011; revised manuscript received 27 March 2012; published 13 June 2012)

\begin{abstract}
We present measurements of the superconducting gap of $\mathrm{URu}_{2} \mathrm{Si}_{2}$ made with scanning tunneling microscopy using a superconducting tip of Al. We find tunneling conductance curves with a finite value at the Fermi level. The density of states is V shaped at low energies, and the quasiparticle peaks are located at values close to the expected superconducting gap from weak-coupling BCS theory. Our results point to rather opened gap structures and gap nodes on the Fermi surface.
\end{abstract}

DOI: 10.1103/PhysRevB.85.214512

PACS number(s): 74.70.Tx, 71.27.+a, 74.25.Jb, 74.55.+v

\section{INTRODUCTION}

Superconductivity emerges out of heavy fermions in a number of materials. ${ }^{1,2}$ Yet some of the basic fundamental properties of heavy-fermion superconductors remain uncharacterized. Results from macroscopic measurements such as specific heat or thermal conductivity imply that the superconducting gap has zeros in some parts of the Fermi surface, forming the much discussed line or point nodes characteristic of unconventional or reduced symmetry superconductivity. ${ }^{3}$ Unconventional superconductivity is indeed likely to be favored within strongly correlated heavy electrons, to avoid mutual electron repulsion in the formation of Cooper pairs.

Recently, the application of the scanning tunneling microscopy (STM) technique to heavy fermions has brought the field a significant step further. ${ }^{4-11}$ Attention has been turned to the so-called hidden order state of $\mathrm{URu}_{2} \mathrm{Si}_{2}$, with the synthesis of a new generation of high-quality ultraclean samples in this compound. ${ }^{12}$ The low-temperature hidden order (HO) phase of $\mathrm{URu}_{2} \mathrm{Si}_{2}$ is indeed characterized by a low carrier density and huge entropy changes with a microscopic ordering whose nature is not yet determined. ${ }^{13}$ If this phase were antiferromagnetically ordered, the moment magnitude would be far too small $\left(0.02 \mu_{B}\right)$ to account for the large entropy changes. Thus, antiferromagnetism (AF) is believed to be not an intrinsic phenomena ${ }^{14}$ but an extrinsic residual component due to the proximity of AF induced at rather low pressure $(0.5 \mathrm{GPa})$ or uniaxial stress. ${ }^{15-17}$ More complex multipole ordering has been proposed: octupole, ${ }^{18}$ hexadecapole,,${ }^{4,19}$ and dotriaecutapole ${ }^{20}$ with even a nematic character. ${ }^{21}$ Helical Pomeranchuk order, ${ }^{22}$ a modulated spin liquid, ${ }^{23}$ and a hybridization wave ${ }^{24}$ have been considered. Early hints towards quadrupolar ordering have been more recently excluded using detailed experiments. ${ }^{25,26}$ The evolution of a gap opening when entering the low-temperature $\mathrm{HO}$ phase at $T_{H O}=17.5 \mathrm{~K}$ has been followed in detail in atomically resolved experiments. ${ }^{4-7}$ Hybridization between heavy quasiparticles as viewed from scattering in Th-doped samples has been discussed in terms of interference effects between multiple-channel tunneling. ${ }^{27,28}$

This peculiar ground state hosts a superconducting phase inside, whose properties are ill known, in spite of much work since its discovery in $1985 .^{29}$ Thus, the simple characterization of the superconducting state remains of great importance. ${ }^{15,16,30,31}$ The presence of nodes in the supercon- ducting gap seems well established from several macroscopic experiments, such as specific heat or thermal conductivity. ${ }^{32-35}$ Evidence for multiple gaps has been provided notably by the upper superconducting critical field. ${ }^{24,36}$ This is not very surprising, as the band structure is complex with sheets showing different mass renormalizations. ${ }^{31,37}$ The measurement of the tunneling spectroscopy of the superconducting density of states has eluded until now all experimental attacks, although early point contact experiments yielded some insight. ${ }^{38,39}$ Here we provide successful tunneling spectroscopy results in the superconducting phase. We determine values for the superconducting gap and its temperature dependence, and find a density of states, which changes as a function of the position and has a finite value at the Fermi level.

\section{EXPERIMENTAL}

We use a STM device in a MX400 dilution refrigerator of Oxford Instruments built and tested following previous work. ${ }^{40,41}$ We use superconducting tips of $\mathrm{Al}^{42,43}$ These tips allow investigating, at the same time, $\mathrm{S}-\mathrm{URu}_{2} \mathrm{Si}_{2}$ and $\mathrm{N}-\mathrm{URu}_{2} \mathrm{Si}_{2}$ tunneling curves by measuring, respectively, at zero field and with a small magnetic field above the critical field of the Al tip (of order of $0.04 \mathrm{~T}$; see Ref. 43). The device features a positioning system which allows changing the scanning window of $4 \mu \mathrm{m}^{2}$ in situ, and bringing the tip to a sample of the same material for cleaning and preparation. We measure the tunneling current $I$ across the junction while applying a bias voltage ramp $V$. The tunneling conductance curves $d I / d V$ versus bias voltage are obtained by numerical differentiation and are usually normalized to the conductance obtained at a bias voltage a few times above the voltage where superconducting features appear. Superconducting tips of $\mathrm{Al}$ are prepared and cleaned in situ on an $\mathrm{Al}$ pad by mechanical annealing from repeated indentation. The scheme of the experiment is presented in Fig. 1(a). The tip is cleaned through an initial repeated indentation process, i.e., by inserting the tip into the sample and then making indentation and retraction repeatedly with an amplitude of some hundreds of nm using the z-piezo. When the tip is pulled out, after some thousands of repeated indentations, we observe clean conductance versus displacement curves which identify few-atom Al contacts [see Fig. 1(b)]. ${ }^{42,43}$ The tip is totally pulled out of the $\mathrm{Al}$ sample, and we observe superconducting S-S tunneling curves 
(a)
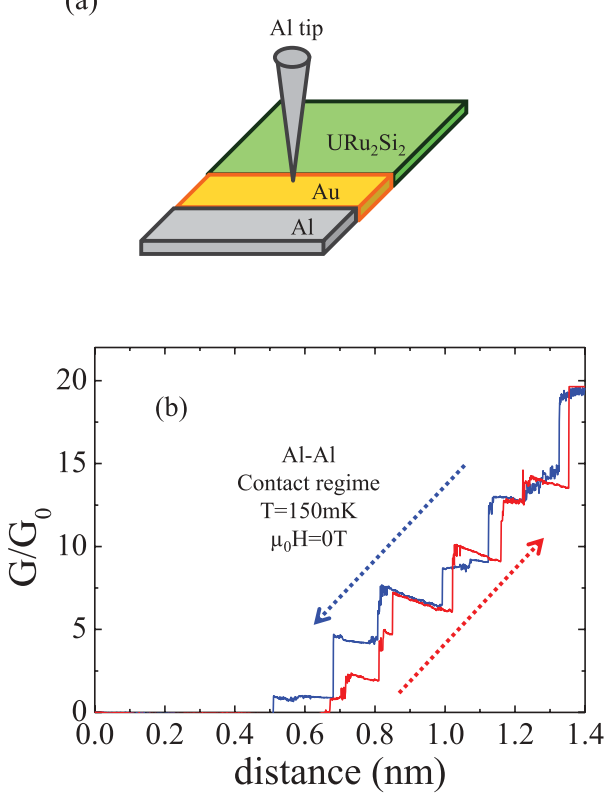

(c)

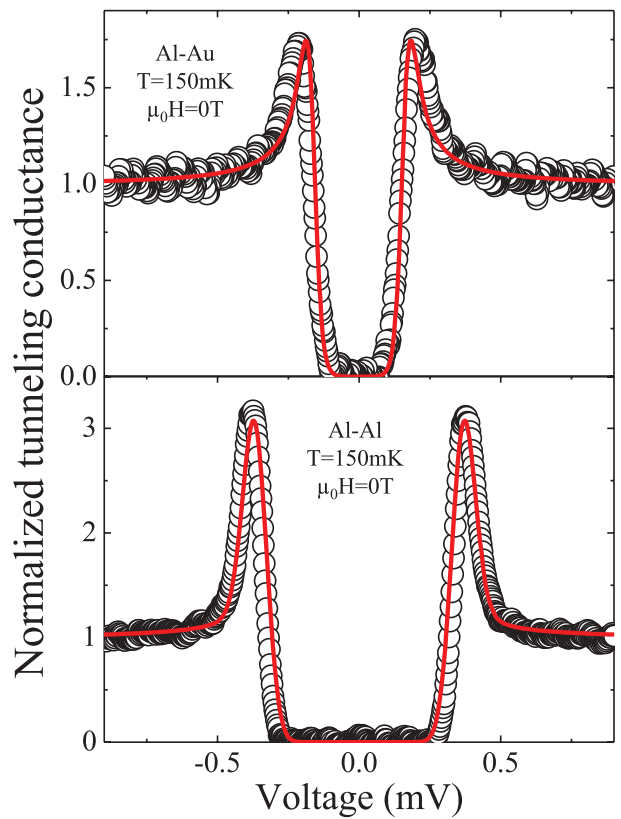

FIG. 1. (Color online) (a) Scheme of the experiment. (b) Conductance vs displacement curves when retracting the tip after a continued repeated indentation procedure made following Refs. 42,43. Curves during indentation (blue upper curve) and tip retraction (red lower curve) are highlighted by arrows. The conductance shows the characteristic Al-Al single-atom point contact features, namely steps at single- and few-atom contacts at values related to the quantum of conductance $G_{0}=2 e^{2} / h$ with a negative slope as plotted here (see Ref. 42 and references therein). The work function in the tunneling limit (at $G \ll G_{0}$, i.e., well below $1 \mu \mathrm{S}$ ), is of several $\mathrm{eV}$. The whole curve shows that the last atoms are of $\mathrm{Al}$ and that the tip can be used for clean vacuum tunneling. (c) When the tip is separated from the sample, clean $\mathrm{Al}-\mathrm{Al}$ spectra are observed. S-S curves (bottom panel) are taken when the Al tip is located above the Al sample. N-S curves are obtained when the same tip is moved in situ on top of an Au sample (top panel). Red lines are fits to BCS using $\Delta=0.16 \mathrm{meV}, T=150 \mathrm{mK}$, and an energy resolution (bias voltage jitter) of $26 \mu \mathrm{eV}$.

in the current versus bias voltage curves. In clean $s$-wave BCS $\mathrm{S}$-S tunnel junctions, the simplest $s$-wave BCS theory gives divergent quasiparticle peaks in the conductance. The width of the actually measured quasiparticle peaks can thus be associated with the voltage jitter of the experiment, ${ }^{40-44}$ for which we find here $26 \mu \mathrm{eV}$ [Fig. 1(c), lower panel]. Once the tip has been thus characterized and the associated conductance verified to follow expected S-S features, it is moved to a clean Au sample. The N-S tunneling conductance gives then a good measurement of the lowest temperature attained in the microscope. ${ }^{45}$ Simple BCS $s$-wave fits of the experimental conductance curve [Fig. 1(c), upper panel] give $150 \mathrm{mK}$. Finally, the tip is brought to the sample of $\mathrm{URu}_{2} \mathrm{Si}_{2}$ for measurement.

Single crystals of $\mathrm{URu}_{2} \mathrm{Si}_{2}$ were grown by the Czochralski method in a tetra-arc furnace with argon gas atmosphere according to Ref. 12 . We decided to break $\mathrm{URu}_{2} \mathrm{Si}_{2}$ samples along the basal plane of the tetragonal structure at room temperature ambient conditions immediately before mounting them on the STM and cooling down. This has the disadvantage that surface contamination cannot be totally avoided, but it also allows the use of a setup suitable for superconducting tips, where the procedures described above can be performed. Moreover, the surface can be optically inspected prior to cool down and samples with nice looking surfaces can be chosen. Many materials prepared ex situ have been found to show clean tunneling features and bulk related spectroscopic curves with a high reproducibility. Successful experiments have been made in ex situ cleaved, as grown, and even chemically etched surfaces. ${ }^{9,11,25,46,47}$ Additionally, in our setup we can macroscopically change at the lowest temperatures the scanning window without heating. ${ }^{40}$ This allows searching in situ over the whole mm-sized sample the scanning windows (of $4 \mu \mathrm{m}^{2}$ ) with best tunneling conditions. In this way, we could find in $\mathrm{URu}_{2} \mathrm{Si}_{2}$ scanning windows with high work functions and a clean surface providing images which are independent of the value of the tunneling current. There, we often found irregular surfaces, within which we could find small atomically flat areas. An example of the topography is shown in Fig. 2. The atomic lattice of the tetragonal basal plane can be resolved [see Fig. 2(b)], with height modulations whose period corresponds to the expected basal plane lattice parameter. We observe high bias voltage Fano-like features [inset of Fig. 2(a)] due to interference between tunneling into light and heavy mass bands, characteristic of heavy fermions. These features appear above some $\mathrm{mV}$ and are comparable to those obtained previously. ${ }^{4-7,10}$ In this work we focus on tunneling spectroscopy measurements at low bias voltage in order to explore the superconducting phase of $\mathrm{URu}_{2} \mathrm{Si}_{2}$. The typical junction conductance is of $0.4 \mu \mathrm{S}$ and the bias voltage of about $1.25 \mathrm{mV}$. The tunneling curves at zero field show clean $\mathrm{S}-\mathrm{S}^{\prime}$ tunneling features, which go over into $\mathrm{N}-\mathrm{S}^{\prime}$ features when applying a magnetic field of $0.1 \mathrm{~T}$.

\section{RESULTS AND DISCUSSION}

In Fig. 3 we show some characteristic tunneling spectroscopy curves obtained at different positions at zero magnetic field $\left(\mathrm{S}-\mathrm{URu}_{2} \mathrm{Si}_{2}\right)$. The superconducting features of $\mathrm{URu}_{2} \mathrm{Si}_{2}$ 


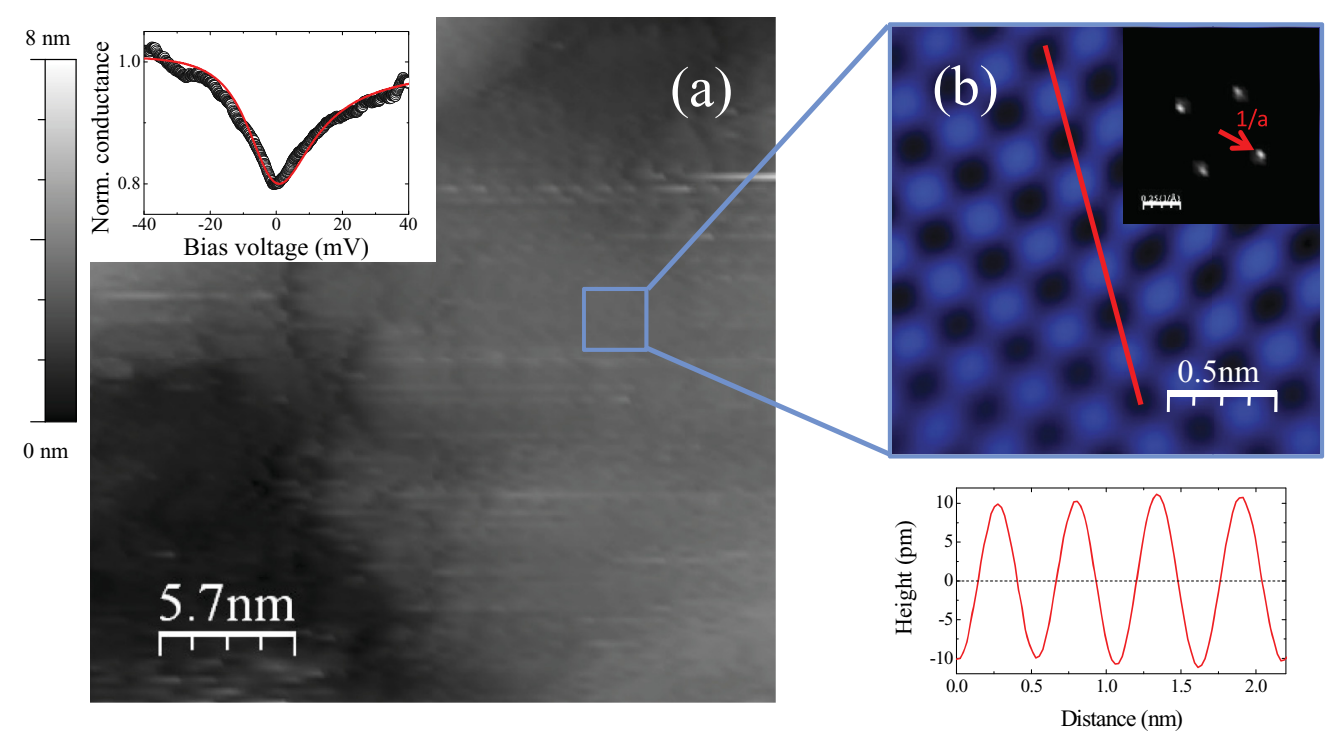

FIG. 2. (Color online) (a) Example of the topography observed, showing typical roughness of about $8 \mathrm{~nm}$. The image was taken at a conductance of $0.4 \mu \mathrm{S}$ and a bias voltage of $1.25 \mathrm{mV}$. In these regions, small flat areas can be found, where atomic resolution can be obtained. Such regions show Fano-like features in the high bias voltage conductance, resembling those obtained in previous work over a terminated $\mathrm{Ru}$ surface (inset: the red line is a fit to a Fano function using an asymmetry parameter $q=-0.2$ and a width of 12 meV) (Refs. 4-7). (b) shows an atomic resolution image taken at a conductance of $0.27 \mu \mathrm{S}$ and a bias voltage of $1.87 \mathrm{mV}$. It shows the basal plane of the tetragonal structure, possibly of the Ru sublattice (Refs. 4-7), and has been Fourier filtered to reveal salient features at the reciprocal lattice wave vector of $0.03 \mathrm{~nm}^{-1}$. From the line scan in real space shown in the bottom panel, we obtain a lattice parameter of $0.3 \mathrm{~nm}$, possibly corresponding to the $\mathrm{Ru}$ interatomic distance.

are clearly resolved in them. These features are homogeneous over small flat areas, but, in different positions, we find slight differences, as presented in Fig. 3. They appear to be related to the particular area where they were taken, and they change at scales of about $10 \mathrm{~nm}$. In the $\mathrm{S}-\mathrm{URu}_{2} \mathrm{Si}_{2}$ normalized tunneling conductance curves [Fig. 3(a), bottom panels], we observe zero conductance at the Fermi level, resulting from the zero density of states of Al, which increases steeply above $0.2 \mathrm{mV}$, showing a pronounced shoulder. $\mathrm{S}-\mathrm{S}^{\prime}$ conductance curves at very low temperatures between two $s$-wave BCS superconductors have a zero conductance up to the sum of both gaps, where a steep peak is found [Fig. 1(b) and Refs. 48-50]. The presence of a marked shoulder in our experiments shows that the density of states of $\mathrm{URu}_{2} \mathrm{Si}_{2}$ is different from a conventional single-gap $s$-wave superconductor.

The tunneling conductance between a superconducting tip and a sample is, in most simple single-particle models, given by $I(V) \propto \int d E\left[f_{T}(E-e V)-f_{S}(E)\right] N_{T}(E-e V) N_{S}(E)$, where $N_{T}(E)$ and $N_{S}(E)$ are the respective densities of states of tip and sample, and $f_{T, S}$ the respective Fermi occupation functions. Using previously determined $N_{T}(E)$ from the curves obtained when the tip is on a normal Au sample [Fig. 1(c), upper panel], ${ }^{42,43}$ we can obtain $N_{S}(E)$ by deconvolution from the integral, getting the curves shown in Fig. 3(b). The deconvoluted curve is of course smeared and does not show noise fluctuations of the experimental conductance. ${ }^{51}$ Note the peculiar low-energy behavior, with a V-shaped form at low energies and well-developed quasiparticle peaks. This $\mathrm{V}$ shape actually produces the shoulder observed in the tunneling conductance, and shows a continuous increase of the density of states from zero energy, as expected in a superconductor with nodes in the gap function. The low but finite zero energy density of states and its changes as a function of the position can be related to band or orientation dependent tunneling into zero gap regions. The topographic features of the surface can also influence the results. More detailed measurements in large, atomically resolved areas will be helpful to explore this further.

Application of $0.1 \mathrm{~T}$ drives the tip into the normal state and reveals a tunneling density of states characterized by a low but finite value close to zero energy and wide quasiparticle peaks, pointing towards a sizable distribution of values of the superconducting gap (see Fig. 4). Previous reported values of the superconducting gap from tunneling spectroscopy give gap sizes several times $\Delta_{0}=1.73 k_{B} T_{c} \cdot{ }^{38,39}$ Here, instead, we observe quasiparticle peaks located roughly at $0.30 \mathrm{meV}$, which is $1.36 \Delta_{0}$. All this is in good agreement with the reduced jump of the specific heat $\frac{\Delta C}{\gamma T_{c}} \sim 1$ derived by entropy conservation. $^{12}$

The temperature dependence of $\mathrm{N}-\mathrm{URu}_{2} \mathrm{Si}_{2}$ curves is shown in Fig. 5. Superconducting features of $\mathrm{URu}_{2} \mathrm{Si}_{2}$ disappear around $1.5 \mathrm{~K}$. We can plot the temperature dependence of the density of states at the Fermi level, and of the energy for which the derivative of the density of states is maximum. Within experimental uncertainty, the latter follows roughly simple BCS theory. In the former, we do not find an appreciable temperature dependence within the uncertainty of our method. Accuracy in its determination and the scatter found over different positions does not allow us to distinguish the small difference expected between the temperature dependence of the gap value, $\Delta$, in a nodal superconductor and in the most simple isotropic $s$-wave BCS superconductor. ${ }^{52}$ 
(a)

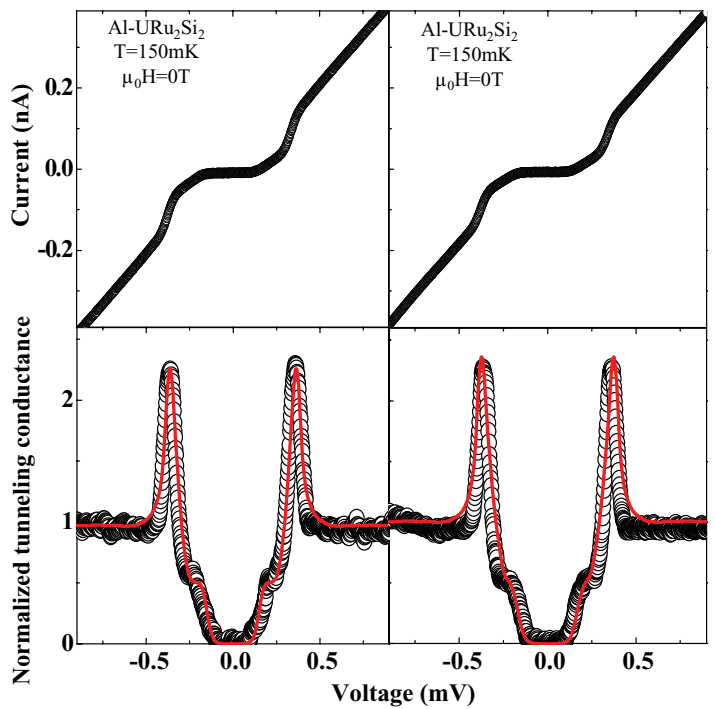

(b)

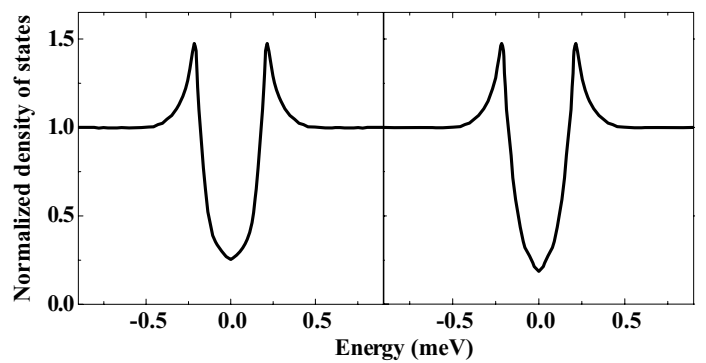

FIG. 3. (Color online) (a) We show tunneling current vs bias voltage (upper panels) and normalized tunneling conductance (lower panels) curves obtained at $0.15 \mathrm{~K}$ and zero field, with the Al tip being superconducting. Red lines in (a) are convolutions obtained using the density of states of $\mathrm{Al}$ for the tip and the normalized density of states shown in (b) for $\mathrm{URu}_{2} \mathrm{Si}_{2}$. Curves are obtained at two different positions of the surface.

Note that we find a small difference between the densities of states generally found using superconducting tips at zero field and those found using normal tips at $0.1 \mathrm{~T}$. Remarkably, when the tip is normal, the quasiparticle peaks are more rounded and located at $20 \%$ higher energies and an amount of states close to the Fermi level is decreased by $10 \%$, leading, in some particular positions, to apparently better developed BCS-like curves. Interestingly, orbital pair breaking effects by the magnetic field, such as the ones produced by the presence of vortices close to the tip, should lead to a decrease in the size of the gap and an increase in the Fermi level conductance, i.e., opposite to what is observed. Thus, either paramagnetic effects appear in the density of states, or the nodes tend to close and the gap opens in the presence of a magnetic field.

Note also that the method for obtaining the density of states discussed in Figs. 3-5 is the simplest approximation for tunneling and assumes single-particle tunneling. In a strongly correlated heavy fermion, simultaneous tunneling into light and heavy masses, as well as Fermi liquid effects, can lead to substantial modifications of the tunneling spectra, as mentioned previously and discussed in Refs. 4-7,27,28,53 in relation with measurement at energies above the meV. It is not yet clear how these features may influence superconducting tunneling at smaller energy scales. A more detailed atomic (a)

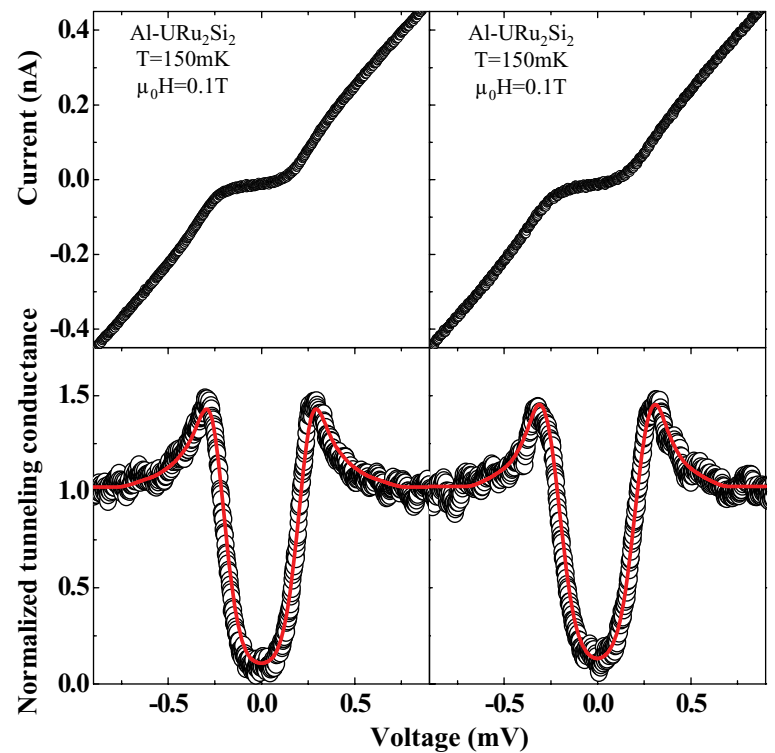

(b)

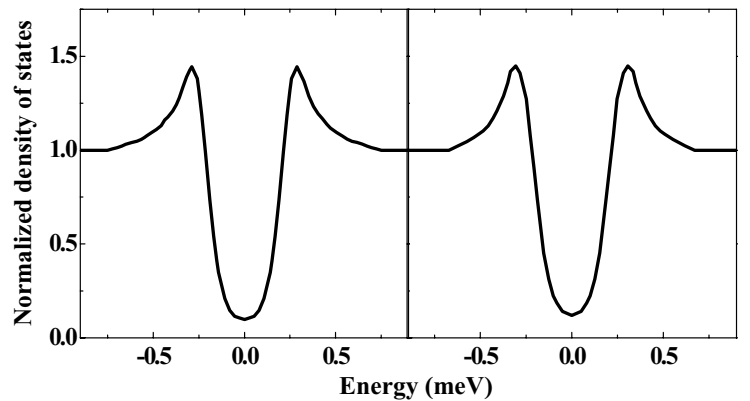

FIG. 4. (Color online) (a) Tunneling current vs bias voltage (upper panels) and normalized tunneling conductance curves (lower panels) obtained at $0.15 \mathrm{~K}$ by applying a magnetic field of $0.1 \mathrm{~T}$, which drives the Al tip to the normal state. Red lines in lower panels of (a) show the tunneling conductance obtained using the normalized density of states shown in (b). Curves are obtained at two different points on the surface.

size study simultaneously imaging high bias voltage and superconducting features would give more insight. Additionally, a theory of $S-S^{\prime}$ tunneling where $S^{\prime}$ is a heavy fermion may help to establish a more direct relationship between the superconducting order parameter and the features observed here in the density of states, namely, V shape at low energies, finite value at zero energy, and slight opening when applying a magnetic field.

Other features characteristic of ${\mathrm{S}-\mathrm{S}^{\prime}}^{\prime}$ junctions, such as the Josephson effect and the temperature dependence of the $\mathrm{S}-\mathrm{S}^{\prime}$ conductance curves, require a detailed analysis and more experiments. Note that the Josephson coupling energy at the tunneling conductance used here is far below the thermal energy $k_{B} T$, which brings the Josephson peak below the resolution of the current measurement, even in an Al S-S junction. ${ }^{42}$ Measurements at a lower tunneling conductance and as a function of temperature are under way.

Finally, let us remark that often specific-heat data show a transition centered around 1.3-1.4 K, which is broad and strongly featured. ${ }^{12,54}$ The specific heat increases well above the midpoint of the transition, evidencing a reduced entropy 
(a)

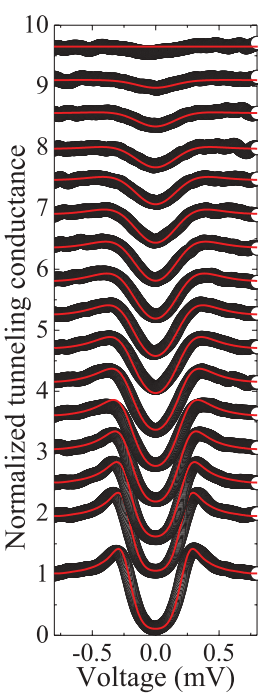

(b)

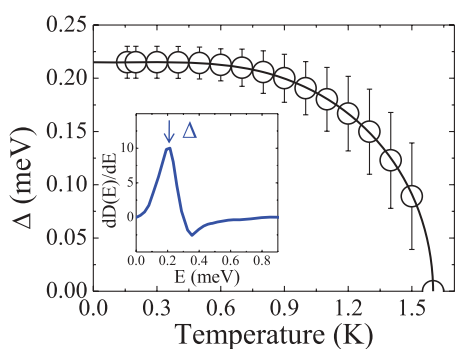

FIG. 5. (Color online) The temperature dependence of the tunneling spectroscopy of $\mathrm{URu}_{2} \mathrm{Si}_{2}$ at $0.1 \mathrm{~T}$ is shown in (a). The data are taken, from bottom to top, at $0.15 \mathrm{~K}$ and from 0.2 to $1.6 \mathrm{~K}$ at steps of $0.1 \mathrm{~K}$. At each temperature, we deconvolute the density of states, and make its derivative as a function of energy, shown as a blue line for $0.15 \mathrm{~K}$ in the inset of (b). The inflexion point in the quasiparticle peak of the density of states is shown by an arrow, and can be taken as a good measurement of the superconducting gap. Its temperature dependence is shown in (b). The black line is a guide to the eye. The error bars highlight the uncertainty in the determination of the deconvolution, which increases together with temperature smearing. Red lines in (a) are the conductance curves obtained using the deconvoluted density of states (Ref. 51).

at temperatures close to or above $1.5 \mathrm{~K}$. The origin of such a featured transition has remained ill understood. On the other hand, when applying pressure, the resistivity retains superconducting features above $\approx 0.7 \mathrm{GPa}$, where antiferromagnetism appears, ${ }^{16}$ but no bulk superconductivity is observed in the specific heat above this pressure. Our results show that the conductance remains gapped up to $1.5 \mathrm{~K}$, and that the density of

states changes in different points on the surface. Remarkably, the residual term in the specific heat is small, of the order of $10 \%$, agreeing with the observed low values of the zero energy conductance observed here. Thus, the superconducting behavior appears to be inhomogeneous, without a significant pair breaking effect affecting the low energy density of states. On the other hand, evidence for an anomalously low carrier density has been provided, and related to the hidden order gap opening. ${ }^{55}$ The low carrier density can make the superconducting properties sensitive to structural distortion. A complex order parameter, such as, e.g., the suggestion of a "chiral" state governed by the nematic HO phase, ${ }^{35}$ could also lead to inhomogeneous superconducting behavior.

\section{CONCLUSIONS}

In summary, we have measured the tunneling spectroscopy in the superconducting phase of $\mathrm{URu}_{2} \mathrm{Si}_{2}$ using very low temperature scanning tunneling spectroscopy. Our results are a significant instrumental step forward which will help to understand properties of heavy-fermion superconductors. Although we find rather irregular surfaces, tunneling curves show opened gap structures, with values of the order of the weak-coupling gap $\left(1.73 k_{B} T_{c}\right)$ and a small but finite density of states at the Fermi level. Finally, the finite and V-shaped density of states observed close to the Fermi level, most clearly revealed when using a superconducting tip at zero field, points towards the presence of nodes along some directions of the Fermi surface.

\section{ACKNOWLEDGMENTS}

The Laboratorio de Bajas Temperaturas is associated with the ICMM of the CSIC. This work was supported by the Spanish MICINN and MEC (Consolider Ingenio Molecular Nanoscience CSD2007-00010 program, FIS201123488, ACI-2009-0905, and FPU grant), by the Comunidad de Madrid through program Nanobiomagnet, and by ERC (NewHeavyFermion) and French ANR projects (CORMAT, SINUS, DELICE). *hermann.suderow@uam.es

${ }^{1}$ J. Flouquet, Prog. Low Temp. Phys. 15, 139 (2005).

${ }^{2}$ C. Pfleiderer, Rev. Mod. Phys. 81, 1551 (2009).

${ }^{3} \mathrm{~V}$. Mineev and T. Samokhin, Introduction to Unconventional Superconductivity (Gordon and Breach SV Publishers, New York, 1999).

${ }^{4}$ K. Haule and G. Kotliar, Nat. Phys. 5, 796 (2009).

${ }^{5}$ A. Schmidt, M. Hamidian, P. Wahl, F. Meier, A. Balatsky, J. Garrett,

T. Williams, and G. Luke, Nature (London) 465, 570 (2010).

${ }^{6}$ P. Aynajian, E. da Silva Neto, C. Parger, Y. Huang, A. Pasupathy, J. Mydosh, and A. Yazdani, Proc. Natl. Acad. Sci. USA 107, 10383 (2010).

${ }^{7}$ M. Hamidian, A. Schmidt, I. Firmo, M. Allan, P. Bradley, J. Garrett, T. Williams, G. Luke, Y. Dubi, A. Balatsky et al., Proc. Natl. Acad. Sci. USA 108, 18233 (2011).

${ }^{8}$ H. Suderow, S. Vieira, J. D. Strand, S. Bud'ko, and P. C. Canfield, Phys. Rev. B 69, 060504(R) (2004).
${ }^{9}$ H. Suderow, K. Behnia, I. Guillamon, V. Crespo, S. Vieira, D. Kikuchi, Y. Aoki, H. Sugawara, and H. Sato, Phys. Rev. B 77, 153101 (2008).

${ }^{10}$ S. Ernst, S. Wirth, F. Steglich, Z. Fisk, J. L. Sarrao, and J. D. Thompson, Phys. Status Solidi B 247, 624 (2010).

${ }^{11}$ O. Fischer, M. Kugler, I. Maggio-Aprile, Ch. Berthod, and Ch. Renner, Rev. Mod. Phys. 79, 353 (2007).

${ }^{12}$ T. D. Matsuda, E. Hassinger, D. Aoki, V. Taufour, G. Knebel, N. Tateiwa, E. Yamamoto, Y. Haga, Y. Onuki, Z. Fisk et al., J. Phys. Soc. Jpn. 80, 114710 (2011).

${ }^{13}$ J. A. Mydosh and P. M. Oppeneer, Rev. Mod. Phys. 83, 1301 (2011).

${ }^{14}$ K. Matsuda, Y. Kohori, T. Kohara, K. Kuwahara, and H. Amitsuka, Phys. Rev. Lett. 87, 087203 (2001).

${ }^{15}$ H. Amitsuka, K. Matsuda, I. Kawasaki, K. Tenya, M. Yokoyama, C. Sekine, N. Tateiwa, T. C. Kobayashi, S. Kawarazaki, and H. Yoshizawa, J. Magn. Magn. Mater. 310, 214 (2007). 
${ }^{16}$ E. Hassinger, G. Knebel, K. Izawa, P. Lejay, B. Salce, and J. Flouquet, Phys. Rev. B 77, 115117 (2008).

${ }^{17}$ F. Bourdarot, N. Martin, S. Raymond, L.-P. Regnault, D. Aoki, V. Taufour, and J. Flouquet, Phys. Rev. B 84, 184430 (2011).

${ }^{18}$ A. Kiss and P. Fazekas, Phys. Rev. B 71, 054415 (2005).

${ }^{19}$ H. Kusunose and H. Harima, J. Phys. Soc. Jpn. 80, 084702 (2011).

${ }^{20}$ F. Cricchio, F. Bultmark, O. Gránás, and L. Nordstrom, Phys. Rev. Lett. 103, 107202 (2009).

${ }^{21}$ H. Ikeda, M.-T. Suzuki, R. Arita, T. Takimoto, T. Shibauchi, and Y. Matsuda, Nature Physics (2012), doi: 10.1038/nphys2330.

${ }^{22}$ C. M. Varma and L. Zhu, Phys. Rev. Lett. 96, 036405 (2006).

${ }^{23}$ C. Pépin, M. R. Norman, S. Burdin, and A. Ferraz, Phys. Rev. Lett. 106, 106601 (2011).

${ }^{24}$ Y. Dubi and A. V. Balatsky, Phys. Rev. Lett. 106, 086401 (2011).

${ }^{25}$ J. G. Rodrigo, F. Guinea, S. Vieira, and F. G. Aliev, Phys. Rev. B 55, 14318 (1997).

${ }^{26} \mathrm{H}$. Amitsuka, T. Inami, M. Yokoyama, S. Takayama, Y. Ikeda, I. Kawasaki, Y. Homma, H. Hidaka, and T. Yanagisawa, J. Phys.: Conf. Series 200, 012007 (2010).

${ }^{27}$ T. Yuan, J. Figgins, and D. K. Morr, arXiv:1101.2636.

${ }^{28}$ M. Ternes, A. Heinrich, and W. Schneider, J. Phys.: Condens. Matter 21, 053001 (2009).

${ }^{29}$ T. T. M. Palstra, A. A. Menovsky, J. van den Berg, A. J. Dirkmaat, P. H. Kes, G. J. Nieuwenhuys, and J. A. Mydosh, Phys. Rev. Lett. 55, 2727 (1985).

${ }^{30}$ P. Soltan-Panahi, D. Luhmann, J. Struck, P. Windpassinger, and K. Senstock, Nat. Phys. 8, 71 (2011).

${ }^{31}$ P. M. Oppeneer, J. Rusz, S. Elgazzar, M. T. Suzuki, T. Durakiewicz, and J. A. Mydosh, Phys. Rev. B 82, 205103 (2010).

${ }^{32}$ E. Knetsch, J. Petersen, A. Menovsky, M. Meisel, G. Nieuwenhuys, and J. Mydosh, Europhys. Lett. 19, 637 (1992).

${ }^{33}$ J. Brison, P. Lejay, A. Buzdin, and J. Flouquet, Physica C 229, 79 (1994).

${ }^{34}$ K. Yano, T. Sakakibara, T. Tayama, M. Yokoyama, H. Amitsuka, Y. Homma, P. Miranovic, M. Ichioka, Y. Tsutsumi, and K. Machida, Phys. Rev. Lett. 100, 017004 (2008).

${ }^{35}$ Y. Kasahara, H. Shishido, T. Shibauchi, Y. Haga, T. Matsuda, Y. Onuki, and Y. Matsuda, New J. Phys. 11, 055601 (2009).

${ }^{36}$ J. P. Brison, N. Keller, A. Vernière, P. Lejay, L. Schmidt, A. Buzdin, J. Flouquet, S. R. Julian, and G. G. Lonzarich, Physica C 250, 128 (1995).
${ }^{37}$ Y. J. Jo, L. Balicas, C. Capan, K. Behnia, P. Lejay, J. Flouquet, J. A. Mydosh, and P. Schlottman, Phys. Rev. Lett. 98, 166404 (2007).

${ }^{38}$ K. Hasselbach, J. R. Kirtley, and P. Lejay, Phys. Rev. B 46, 5826 (1992).

${ }^{39}$ Y. Naidyuk, H. Lohneysen, G. Goll, I. Yanson, and A. Menovsky, Europhys. Lett. 33, 557 (1996).

${ }^{40}$ H. Suderow, I. Guillamon, and S. Vieira, Rev. Sci. Instrum. 82, 033711 (2011).

${ }^{41}$ M. Crespo, H. Suderow, S. Vieira, S. Bud'ko, and P. C. Canfield, Phys. Rev. Lett. 96, 027003 (2006).

${ }^{42}$ J. G. Rodrigo, H. Suderow, and S. Vieira, Eur. Phys. J. B 40, 483 (2004).

${ }^{43}$ I. Guillamon, H. Suderow, S. Vieira, and P. Rodiere, Physica C 468, 537 (2008).

${ }^{44}$ A. Maldonado, I. Guillamon, H. Suderow, and S. Vieira, Rev. Sci. Instrum. 82, 073710 (2011).

${ }^{45}$ A. Maldonado, H. Suderow, and S. Vieira, Cryogenics 50, 397 (2010).

${ }^{46}$ I. Maggio-Aprile, Ch. Renner, A. Erb, E. Walker, and O. Fischer, Phys. Rev. Lett. 75, 2754 (1995).

${ }^{47}$ Y. De Wilde, M. Iavarone, U. Welp, V. Metlushko, A. E. Koshelev, I. Aranson, G. W. Crabtree, and P. C. Canfield, Phys. Rev. Lett. 78, 4273 (1997).

${ }^{48}$ E. Wolf, Principles of Electron Tunneling Spectroscopy (Oxford University Press, Oxford, 1989).

${ }^{49}$ H. Suderow, E. Bascones, A. Izquierdo, F. Guinea, and S. Vieira, Phys. Rev. B 65, 100519(R) (2002).

${ }^{50}$ J. G. Rodrigo, H. Suderow, S. Vieira, E. Bascones, and F. Guinea, J. Phys.: Condens. Matter 16, R1151 (2004).

${ }^{51}$ I. Guillamon, H. Suderow, S. Vieira, L. Cario, P. Diener, and P. Rodiere, Phys. Rev. Lett. 101, 166407 (2008).

${ }^{52}$ H. Won and K. Maki, Phys. Rev. B 49, 1397 (1994).

${ }^{53}$ A. Yazdani, B. A. Jones, C. P. Lutz, M. F. Crommie, and D. M. Eigler, Science 275, 1767 (1997).

${ }^{54}$ T. Kasahara, T. Iwasawa, H. Shishido, T. Shibauchi, K. Behnia, Y. Haga, T. D. Matsuda, Y. Onuki, M. Sigrist, and Y. Matsuda, Phys. Rev. Lett. 99, 116402 (2007).

${ }^{55}$ K. Behnia, R. Bel, Y. Kasahara, Y. Nakajima, H. Jin, H. Aubin, K. Izawa, Y. Matsuda, J. Flouquet, Y. Haga et al., Phys. Rev. Lett. 94, 156405 (2005). 